\title{
MÉTODO PARA EL ANÁLISIS ESTRATIGRÁFICO DE CONSTRUCCIONES HISTÓRICAS O "LECTURA DE PARAMENTOS"
}

\author{
(A METHOD FOR THE STRATIGRAPHIC ANALYSIS OF HISTORICAL CONSTRUCTIONS \\ OR "FACE READING")
}

\author{
L.Caballero Zoreda,Dr. Arqueólogo \\ Centro de Estudios Históricos-CSIC- Madrid \\ ESPAÑA
}

Fecha de recepción: $13-[1-95$

\begin{abstract}
RESUMEN
El interés del articulo es difundir entre los investigadores y técnicos españoles este método de análisis, desarrollado básicamente por los arquitectos italianos Parenti y Brogiolo. Se basa en la consideración de que el edificio se ha formado por la adición histórica de partes distintas que se someten a las leyes de la estratigrafia y que por ello permiten su lectura según un método arqueológico. Se pretende definir y adecuar su terminologia, sus instrumentos y su proceso de trabajo al lenguaje científico y a la propia experiencia, y exponer algunos de los problemas que plantea.
\end{abstract}

Fecha de recepción. $13-16$

\section{Método de análisis}

Enqué consiste el método.- Con este método se diferencian, ordenan y datan las fases por las que han pasado los edificios hasta llegar a su estadio actual, analizando todos los elementos que los componen y que se les fueron añadiendo históricamente y analizando las distintas actividades y procesos destructivos y constructivos que sufrió. Se trata, por lo tanto, de un método cuya finalidad es básicamente histórica, concretamente la historia de la arquitectura, que contribuye decisivamente a orientar la práctica de la arquitectura restauradora.

No estamos ante un método cerrado con una aplicación rígida. Al contrario, es un método flexible, auxiliar o instrumental, que permite la posibilidad de adecuarse a circunstancias muy variadas. Es importante no olvidar su procedencia, en última instancia de la estratigrafía geológica ${ }^{1}$ y directamente del llamado "método Harris" con el que se aplica ésta a la excavación arqueológica (Harris 1992). El propio Harris recoge ejemplos de lectura de paramentos, adelantándose a su aplicación generalizada a la arquitectura (Harris 1991:90-93 y 1992:96).

\section{Conceptos}

Cómo denominar el método. - Con una simple ojeada a la bibliografía de este método se constata que no existe unanimidad sobre cómo denominarle -método, análisis, investigación, estudioo lectura; arqueológico, estratigráfico, arquitectónico, arqueo-arquitectónico-; ni sobre cómo llamar a su objeto de estudio -edilizia, arquitectura, 
construcción, estructura, muraria, muratura, paramentos, elevati, alzados-

Esta simple relación refleja, además de un problema de denominación, unaindecisión más profunda sobre cómo se entiende y qué se pretende con el propio método. Adjetivar como arqueológico o estratigráfico el análisis de los edificios supone aceptar que se pueda confundir con el método arqueológico por excelencia, la excavación de su subsuelo; afirmar que efectivamente en el edificio hay estratos; y utilizar un término que pertenece a una profesión distinta de la arquitectura. Sin embargo estratigrafia es el término que utilizaParenti, pionero en este método. Es evidente que podrian presentarse argumentos a favor de nombrarle análisis arquitectónico -como hace uno de los trabajos que se incluyen en este número de INFORMES por depender de un departamento administrativo dedicado a la arquitectura y no a la arqueología- y que por este camino llegaríamos al enrevesado término de consenso arqueoarquitectónico. Por todo ello quizás se tiende a sustituir el término análisis arqueológico por lectura esiratigráfica de edificios o más simplemente por lectura de paramentos, que parece que en nuestro lenguaje hablado -todavía no en el escrito- es más cómodo, a pesar de que lectura es un término demasiado lingüístico y amplio, y paramento demasiado concreto y con el que se pierde la unidad contextual del edificio.

Pero el método ha surgido como una aplicación del método estratigráfico arqueológico, según lo define Harris, al estudio de los edifícios históricos. Personalmente prefiero llamar al objeto de estudio construcción, que arquitectónicamente significa la actividad y el efecto de construir y sintácticamente la relación entre las partes -que es fundamental en este método- y además es la palabra española más cercana a la riqueza semántica del edilizia italiano. Para evitar la duda entre arqueologíay arquitectura puede adjetivarse la construcción de histórica, lo que quizás consiga el consenso de unos y otros profesionales. De modo que mi propuesta es la de análisis, estratigráfico o arqueológico, de construcciones históricas.

Los principios estratigráficos. - Podemos preguntarnos si en el edificio se pueden diferenciar elementos que cumplan una función similar a la de los estratos geológicos o arqueológicos, como hace Doglioni (229-232) contestando negativamente. Efectivamente, en una observación superficial parece que, por ejemplo, cuando se restauran los edificios se pueden rehacer los cimientos sin tener por qué demoler las partes altas más antiguas, de modo que se incumple el principio de que los estratos inferiores son más antiguos que los superiores. Nosotros mismos lo hemos dudado al enfatizar las diferencias existentes entre arquitectura y geología (Caballero 1992).

Sin embargo la contestación es afirmativa como se confirma al verificar que los principios de la estratigrafia son perfectamente aplicables a los elementos que han conformado históricamente el edificio (DyH:1.10 ss. y fenómenos generales, $1.15 \mathrm{ss}$.) y ello a pesar de la diferencia sustancial que existe entre geología y arquitectura, esto es que en la Tierra actúan procesos geológicos, mientras que en el edificio tienen una mayor importancia las acciones antrópicas (el yacimiento se sitúa en un término medio, Harris 1991: Introducción). Harris sólo aplica a la arqueología los dos primeros principios (que llama incorrectamente leyes, Harris 1991:31-32, 51-64):

1.-El de superposición, sucesión y continuidad: los elementos de un edificio, como los estratos, se superponen y adosan unos a otros (Parenti 1988a:269), de modo que un elemento superpuesto o adosado a otro es posterior a el y a la inversa, mientras que cada uno de ellos es coetáneo en toda su extensión. Asi se crean sucesiones cronológicas desde el más moderno, en la situación más alta, al más antiguo, en la más baja.

2.-El de horizontalidad original y continuidad lateral. relacionado con el anterior: los elementos se extienden horizontalmente pero de modolimitado, tendiendo a ocupar todo el hueco que encuentran libre o la superficie útil del edificio como si fuera una "cuenca de sedimentacion" (Parenti 1985:57; Marino: 175).

3.-El de las relaciones de cruce o de corte: los elementos también se cortan unos a otros, de modo que los que cortan son posteriores a los cortados. Dabrio y Hernando consideran este principio básico en el establecimiento de las relaciones temporales en estratigrafia y, efectivamente, es el que explica que muchos elementos que cortan o rellenan las partes bajas del edificio sean posteriores a otros de las altas, contradiciendo aparentemente el primer principio.

4.-El de los "fenómenos" de la discontinuidad temporaly la mayor importancia de los hiatos en el registro estratigráfico: en la práctica la seriación de elementos nunca se presenta completa, tanto para una zona dada del edificio como incluso para el edificio entero, de modo que el tiempo representado por los elementos es mucho menor que el no representado por ellos y que corresponde a hiatos.

Estos y otros principios y fenómenos ${ }^{2}$, debidamente matizados, sirven para realizar el análisis o la lectura científica del registro estratigráfico, combinándolos entre sí.

Materiales y elementos estratigráficos.- Los materiales son la parte menor en que se puede disgregar el edificio. Pueden ser constructivos, que construyen los elementos y definen sus características; o decorativos, que facilitan la datación dado que en ellos se refleja mejor la evolución de los estilos, aunque la fecha no es privativa de ellos, ya que con los constructivos también se debe -y de hecho se construyen-tipologías de valor cronológico relativo (Parenti 


\section{PRINCIPIOS DE LA ESTRATIGRAFIA}

- LOS ELEMENTOS SE SUPERPONEN Y SE ADOSAN.

- LOS ELEMENTOS OCUPAN HORIZONTALMENTE TODO EL HUECO LIBRE 0 LA SUPERFICIE ÚTIL.

- LOS ELEMENTOS TAMBIEN SE CORTAN UNOS A OTROS.

- LA SERIACIÓN NUNCA ES COMPLETA. EL TIEMPO REPRESENTADO POR LOS ELEMENTOS ES MENOR QUE EL REPRESENTADO POR LOS HIATOS.

DIAGRAMA CON RELACIONES REDUNDANTES

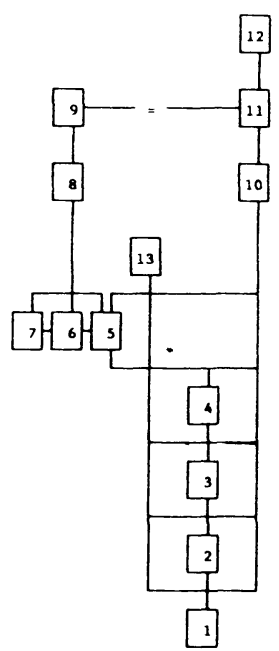

DIAGRAMA REDUCIDO

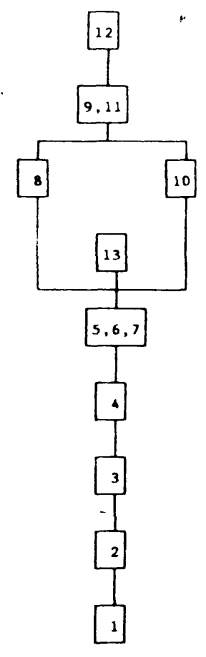

$2,4,6,8$ y 10 son interfaces 2 corta a 1

corta $a, 2$ y

5 cubre a 3 y 1

6 es interfaz de una etapa de obra

5,6 y 7 son iguales

8 corta a 5,6 y 7

9 se adosa a 5, rellena a 7 y es igual a 11

10 corta a $5,4,3,2$ y 1

11 se une a 5, 3 y

12 se adosa a 11

13 cubre a 1,3 y 5
ETAPAS

Iv

1988b). Se puede decir que los materiales constructivos equivalen a la materia que forma sustancialmente los estratos geológicos o los arqueológicos y los materiales decorativos equivalen a los fósiles y las manufacturas cerámicas, vidrios, herramientas, ...- que se encuentran en ellos. El aparejo es la técnica constructiva o manera de utilizar estos materiales para construir, que da lugar a tipologías constructivas con valor cronológico relativo.

El elemento estratigráfico (unidad estratigráfica muraria, USM, para Parenti y Brogiolo; elemento arquitectónico o singular para Coll y otros:97) es la unidad construida menor, individualizable estratigráficamente de las que la rodean, y el objeto fundamental de análisis -un fragmento de muro o de ventana, un mechinal, una pieza del forjado, un relleno, ...- equivalente al estrato geológico o al contexto del yacimiento. Se diferencian elementos verticales-muros-y horizontales -suelos; techos-, aunque estratigráficamente todos funcionan de modo similar. 
Las discontinuidades fisicas y temporales. Superficies $e$ hiatos.- Pero existen elementos que tienen volumen y materialidad y otros que sólo son superficies sin volumen ni materia y que, sin embargo, tienen más valor que los primeros, ya que los delimitan y distinguen entre sí y son portadores de un valor temporal muy superior al de ellos, comovimos que definía el principio 4. Se llaman interfaces ${ }^{3}$, discontinuidades o soluciones de continuidad, límites, superficies o cortes. La comprensión de estas superficies -que también son "elementos" estratigráficos, aunque reservemos este término para los que tienen volumen y materia- es compleja y de hecho falta mucho por avanzar en su sistematización. Como en los elementos se deben diferenciar tres aspectos en las superficies: uno genético, 0 actividad constructiva, que dio lugar a su apariencia geométrica o forma, que a su vez encierra un valor temporal. Geométricamente se definen como los límites o las superficies de los elementos a los que diferencian entre sí, por lo que Harris los considera elementos interfaciales, además de que modelan o definen su forma, lo que en arquitectura tiene gran importancia. Pueden ser producto de acciones positivas, como es la superficie de contacto o la cara originaria de un elemento creado por una acción constructiva, o una superficie de uso como el elemento residual de un suelo (equivalente a la "junta" geológica, DyH:3.10); o bien ser producto de acciones negativas de ruina, destrucción o saneado que llamamos cortes. Normalmente estas superficies son netas, pero pueden estar enmascaradas por enfoscados o tener entre sí un contacto gradual por la reutilización in situ de materiales procedentes de los elementos a que se adosan (DyH:4.3, 8.23). Es muy importante distinguir las superficies de fase o período que separan grupos de elementos unitarios o estructuras (en geología denominadas específicamente "discontinuidades", DyH:9.22-27), de las que diferencian elementos singulares, como las tongadas de etapas de obra.

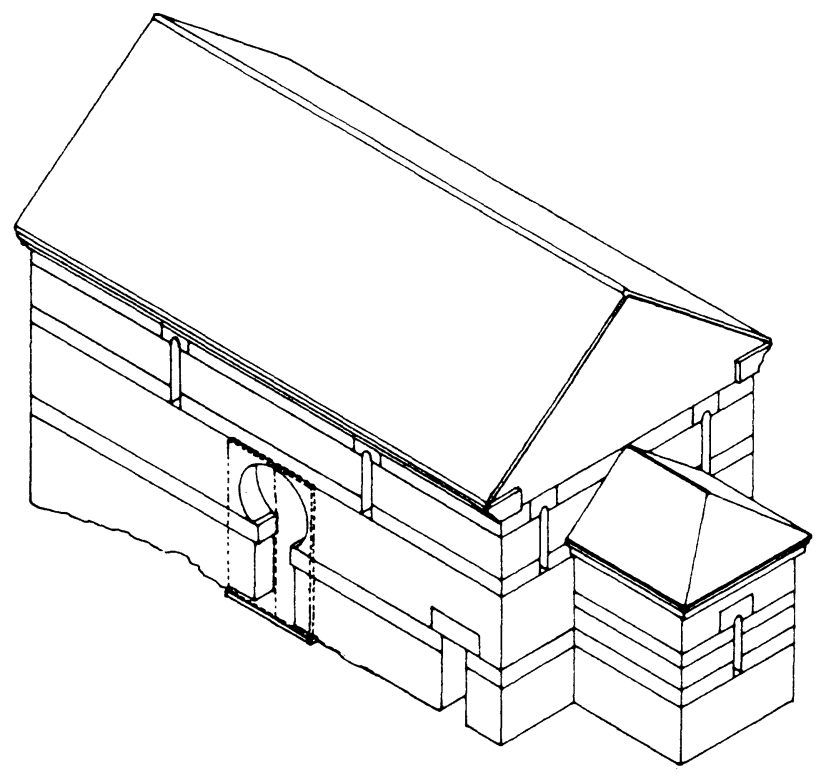

I EDIFICIO PRIMITIVO
Ambos tipos de "elementos", volumétricos y superficiales, son soporte de una cronología concreta. Los elementos normalmente documentan un tiempo corto, el momento en que se construyeron; sus superficies, en cambio, un tiempo largo, por ejemplo el tiempo en que se utilizó como suelo. Pero existen discontinuidades temporales o hiatos que no coinciden con lo documentado por éstos, que suelen corresponder a cortes con los que han desaparecido elementos que documentaban fases y actividades desde entonces no representadas.

Unidades de elementos: estructuray edificio.-Un conjunto de elementos, volumétricos e interfaciales, definidos por su unidad funcional y cronológica-que responden a la misma función y pertenecen a un mismo momento histórico-, suponen una estructura. Una estructura no es un edificio, ni tampoco una de sus partes -una fachada o una nave-, sino la unidad de elementos coetáneos que restan de un edificio histórico, una etapa de la serie estratigráfica que es el edificio. Por lo tanto el edificio, o sea la unidad final llegada a nosotros, estará compuesto por varias estructuras. Lógicamente los elementos que forman una estructura deben tener una similitud de materiales y características -sillería, mampostería estucada-, una unidad temporal -o fase, románico, $2^{\mathrm{a} / 2} \mathrm{~s}$. XIII- y una unidad genética y funcional -constructiva y utilitaria-. Dentro de cada estructura se puedan distinguir etapas de uso -reformas, arreglos, adecuaciones-, que no inciden en la identidad sustancial de la estructura y que sólo definen en ella secuencias secundarias.

\section{EI proceso y los instrumentos de análisis}

Documentación gráfica yobservación.- La documentación gráfica -planimétrica y fotográfica- no es sólo la etapa

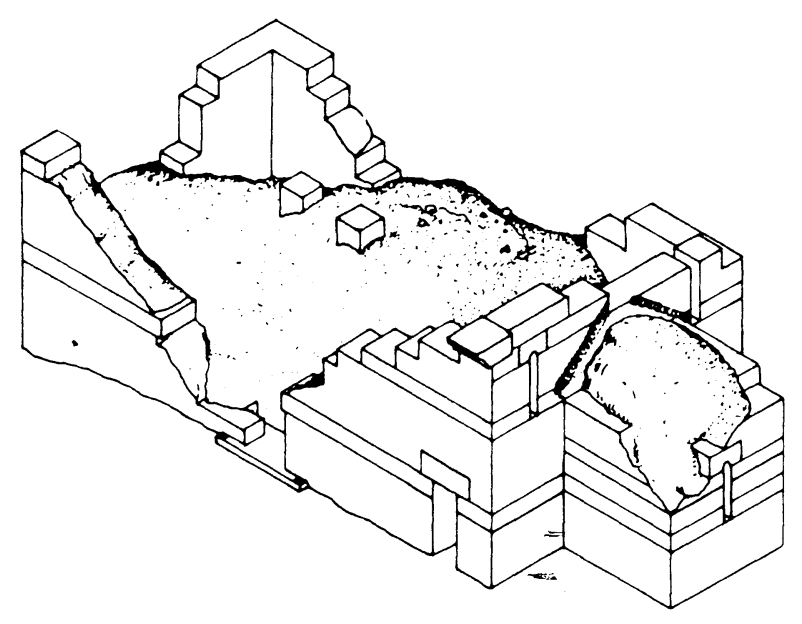

2-RUINA SUPERFICIE DE CORTE 
previa del análisis; se puede considerar su propia esencia. De alguna manera documentar, como sinónimo de leer un documento, supone en el análisis de la construcción histórica lo que la excavación en un yacimiento (frente a la que parece ser la opinión de Doglioni:229-230 y la absurda y errónea de Bonelli. Parenti 1988a:249-250). El análisis diferencial de contextos y la constatación de sus relaciones se basa en la observación visual (Parenti id., lectura personal directa "autoóptica"; también DyH:2.1) que obliga a fijarse con un cierto grado de automatismo que le hace ganar en objetividad. La documentación efectuada por otros o de modo mecánico, no libera de esta labor personal e importantísima.

Cómoreducir el tiempo y los recursos empleados.-Brogiolo (1988a: 15-34 y 1988b: 336-9) propone una graduación de la documentación-grados 0 y A/D- en relación a la rapidez del proceso y a las unidades de referencia documentadas complejo arquitectónico, crujía, alzado general, detalle de alzado, unidad funcional o ambiente, elemento horizontal y elemento arquitectónico ${ }^{4}$. De sus palabras parece deducirse que esta graduación no incide en la calidad de las conclusiones: "Los diversos grados de documentación deben ser proporcionales al interés histórico del complejo arquitectónico examinado y adecuados a la finalidad del análisis (Brogiolo 1988a:34)".

En mi opinión, la calidad de las conclusiones puede ser independiente del grado de documentación e incluso del hecho de analizar todo el edifício o sólo partes escogidas de él, pero nunca de la profundidad con que se realice el análisis. Además su propuesta tiende a confundir las partes históricas del edificio -coincidentes con las fases históricas de la serie estratigráfica- con zonas segregadas artificialmente en el edificio por el analista, con una finalidad instrumental. El análisis de un edificio a nivel de unidades de referencia, tal como él las define, no asegura conclusiones históricas fiables (Parenti 1988a:276). Pero es cierto que se necesitan criterios de economía para usar este método con éxito al analizar grandes edificios (falsa crítica que hace Bonelli:10). Justamente el método estratigráfico ha sido concebido para un sujeto de estudio que rebasa con mucho el volumen de análisis que pueda llegar a representar la arquitectura histórica, como es la geología terrestre. Para reducir significativamenteel tiempo y los recursos empleados en el proceso - pretensión de Brogiolo-, lo lógico es realizar con todo detalle columnas estratigráficas de cortes o tramos previamente elegidos por su aparente mayor riqueza de información estratigráfica, sintetizándolas luego en un diagrama final (un modo similar de "muestreo" propone Parenti 1988a:261y267; comparar con GyH:2.2-4); aunque este análisis parcial no asegura la obtención de la seriación estratigráfica completa, pues es probable que en el resto del edificio no analizado existan relaciones y estratos no representados en 10 analizado, de acuerdo con el principio 4.

Creo no ser parcial al afirmar que la calidad de la documentación no tiene por qué incidir en el grado de validez del resultado final del análisis. Como se comprueba con algunos ejemplos que se presentan aquí, es perfectamente posible realizar análisis suficientemente válidos basados en croquis manuales, sin escala, o realizados en programas informáticos que, en principio, son totalmente inadecuados para la representación gráfica. Es evidente que cuanto más detallada sea la información aportada por el instrumento gráfico documental, más perfecto podrá ser el resultado del análisis; pero puede ocurrir al contrario, el análisis de elementos estratigráficos y la secuencia de un edificio no se resuelve sólo consiguiendo una documentación detallada -y cara-

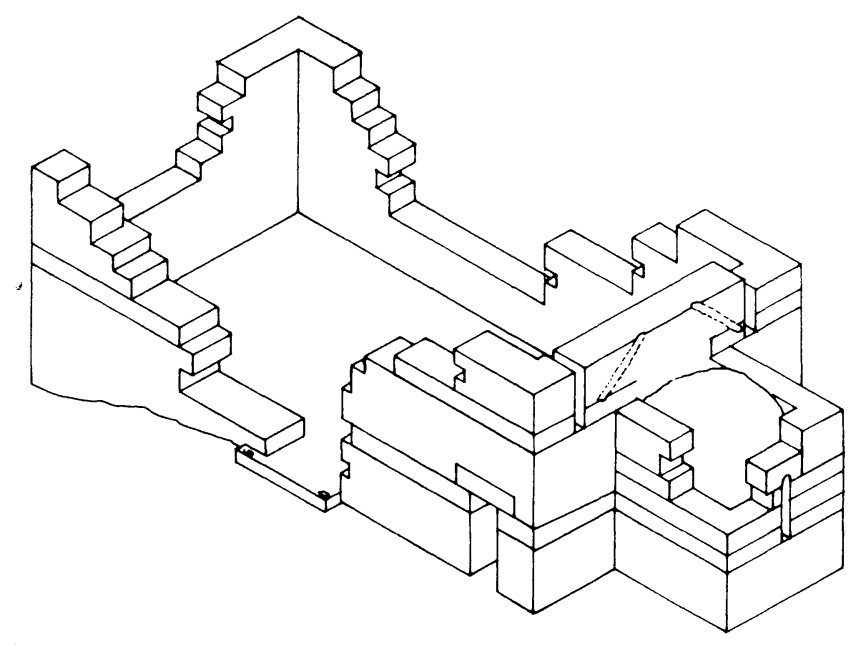

3.- Corte de saneado pReparación para la reconstrucción

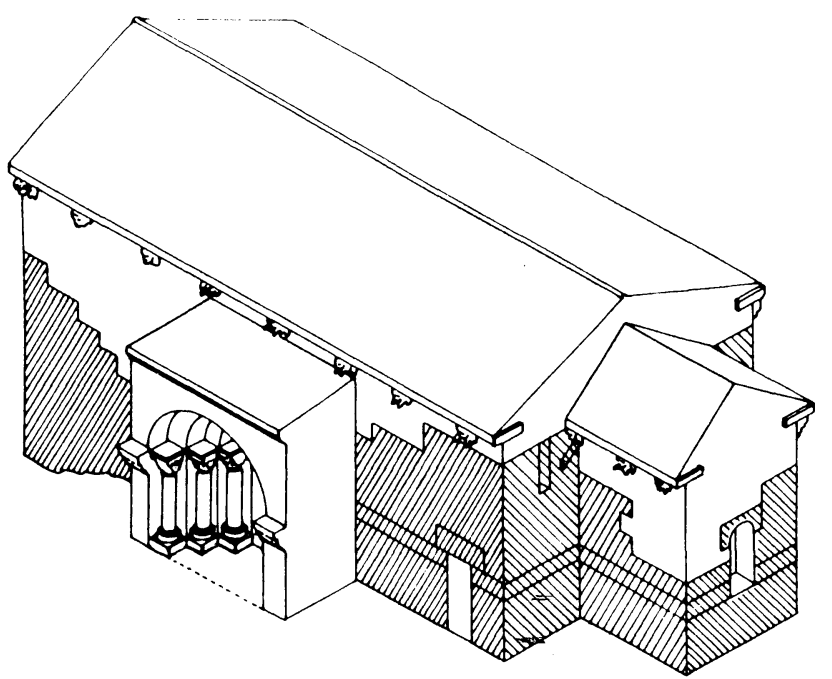

4. estructura anadioa a la primitiva 
División del trabajo.- Efectuada la documentación, el edificio se divide en zonas o cortes auxiliares, que se reparten entre los grupos del equipo. De este modo su volumen se hace abarcable y se facilita el trabajo. Esta división es por tanto arbitraria y auxiliar y es conveniente que abarque tres dimensiones: arriba y abajo, a uno y otro lado y por fuera y dentro del edificio dividido. Las zonas, como he dicho, no deben confundirse con partes estratigráficas o secuenciales del edificio y pueden coincidir o no con las unidades de la documentación planimétrica. Cada zona tendrá su documentación y referencias analíticas correspondientes que deberán luego integrarse en la síntesis final.

La diferenciación de los elementos.- El verdadero análisis comienza con la diferenciación de elementos y superficies. Los criterios para diferenciarlos deben ser estratigráficos y no sólo constructivos (en contra de mi opinión, 1992. Ver Doglioni:231, citando a Manacorda:12). Cada elemento se diferencia por criterios de homogeneidad, individualidad y contemporaneidad, referidos a su forma geométrica, limitada por interfaces o superficies de estratificación, sus componentes y la acción genética o constructiva que la creó, advirtiendo que cada elemento contiene subelementos menores -etapas de obra- (DyH:cap.3).

Las fichas analiticas.- Los elementos e interfaces diferenciados se analizan utilizando fichas donde se describen éstos, las acciones que actuaron desde su creación y las relaciones que poseen con los demás elementos. Las fichas pueden ser de elementos e interfaces y también de estructuras, o sea de unidades históricas.

Cada ficha se debe acomodar al objetivo concreto del trabajo y a las características físicas del edificio que se analiza (Parenti 1988a:255, por ejemplo fichas de revestimientos; Id.:252-254, n.8 fichas oficiales). Pero siempre deberán tener unos campos fijos que son los siguientes: Identificación del instrumento -la ficha- y del elemento o la unidad estratigráfica, a través de un número, su nombre y ubicación. Descripción, composición. Acciones que crearon el elemento o la unidad descrita y sus relaciones con los demás elementos o unidades, en cuadro y diagrama. Interpretación. Referencias.

Problemas concretos de la numeración.- En la práctica la información que tenemos de cada estrato o conjunto de estratos se recupera por el número, a través del cual nos dirigimos a su ficha y a los demás instrumentos que le correspondan. Se usan relaciones numéricas o indices, tanto para evitar duplicaciones de números como para recuperarlos. También, para superar la posible confusión entre los distintos grados estratigráfico/constructivos, utilizamos los millares para identificar los elementos empezando en el 1001- y las centenas para las estructuras
-101 a 999-, dejando las unidades y las decenas para numerar las zonas y otros instrumentos secundarios (Brogiolo propone las centenas para sus elementos arquitectónicos y los millares para las UEM, 1988b:fig. 8).

La norma es que cada ficha debe corresponder a un solo elemento, pero esto en la práctica no es así. Un mismo elemento puede recuperarse por varias fichas con distintos números $\mathrm{y}$, al contrario, por una ficha recuperar varios elementos con el mismo número. El primer caso ocurre cuando la unidad de un elemento se ha perdido, bien por una acción histórica -un muro dividido posteriormente en partes independientes-, bien por los propios criterios de diferenciación ,o porque se decida diferenciar partes ante la duda de que pertenezcan a elementos distintos, para asegurar la certeza del análisis y aunque luego se demuestre que pertenecen al mismo elemento. El segundo caso, cuando los elementos o subelementos se repiten de modo sistemático con exactas características y el análisis inmediato asegura que pertenecen a una misma acción (por ejemplo tongadas de obra o series de mechinales, Parenti, 1988a:261, 267, 268).

El orden de numeración de los elementos plantea una de las diferencias entre la estratigrafía del yacimiento y del edificio. En el yacimiento la numeración coincide con el orden de excavación de los contextos, de modo que, teóricamente, este orden es inverso al de su deposición histórica, principio que de hecho sólo es posible mantener si la superficie a excavar no es demasiado amplia y por lo tanto no se subdivide en distintas series estratigráficas. Si en la práctica de la excavación es difícil mantener este orden, al menos por logística, en el edificio es prácticamente imposible. Lo normal es que sea muy difícil distinguir a primera vista el orden estricto en que se depusieron o construyeron todos y cada uno de los elementos, debido fundamentalmente a la simultaneidad de cada muro que representa una serie estratigráfica individual y a la abundancia de relaciones de corte. Por ello es imposible hacer coincidir el "orden" de numeración con el histórico de deposición, desde el más reciente hasta el más antiguo, como se propone en la excavación. Ello obliga a que la numeración sea aleatoria, siguiendo sólo el orden de nuestro proceso de trabajo (sobre este problema Parenti 1988a:276-7).

Las relaciones estratigráficas y su valor temporal. Los diagramas.- En el cuadro de relaciones de cada ficha, se van anotando las relaciones que se observan entre los elementos. Con estas anotaciones se construye el diagrama $o$ matriz de relaciones, sistema de representación del tiempo, donde los elementos se ordenan cronológicamente según sus relaciones de diacronía, en columnas verticales, y de sincronía, en líneas horizontales. Los diagramas se construyen primero en las fichas, elemento por elemento, para luego irlos uniendo entre sí para crear los de zona. El 
CUADRO DE RELACIONES ESTRATIGRáFICAS

con relación fisica directa (actividades y relaciones temporales):

DIAGRAMA :

\begin{tabular}{||l|l|l|l|l|l|l||}
\cline { 2 - 7 } & unir & apoyar & adosar & cubrir & cortar & rellenar \\
\hline \hline anterior a & & & & & & \\
\hline coetáneo a & & & & & & \\
\hline posterior a & & & & & & \\
\hline
\end{tabular}

sin relación física directa:

igual a,

equivalente $a$,

diagrama final lógicamente debe ser el que reúna los de zona, pero, como luego veremos, puede ocurrir que el proceso de simplificación que supone la diferenciación de estructuras aconseje construir un diagrama final de estructuras y no llegar a uno final de elementos, que por su complejidad puede ser inviable o de difícil comprensión (de ambientes o de paredes para Parenti 1988a:279). Siempre es recomendable construir los diagramas intermedios, pero especialmente es necesario si se decide no construir el diagrama final de elementos, en cuyo caso se debe suplir su ausencia por diagramas de unidades intermedias, bien de zonas o de estructuras -en sus fichas-.

El análisis de las relaciones estratigráfico/constructivas es sin dudala parte más delicada del proceso. En él se mezclan tres lecturas distintas: la de la situación espacial de los elementos -en contacto o no; encima, debajo, al lado- que lleva emparejada la de la acción constructiva que los creó -cubrir, rellenar, apoyar, adosar, cortar, unir, etc. ${ }^{5}-$ que concluye con una secuencia temporal-de coetaneidad o de antero/posterioridad-. Como se ve, las acciones constructivas pueden ser de muchos tipos aunque para facilitar el análisis se reducen a media docena; mientras que las relaciones

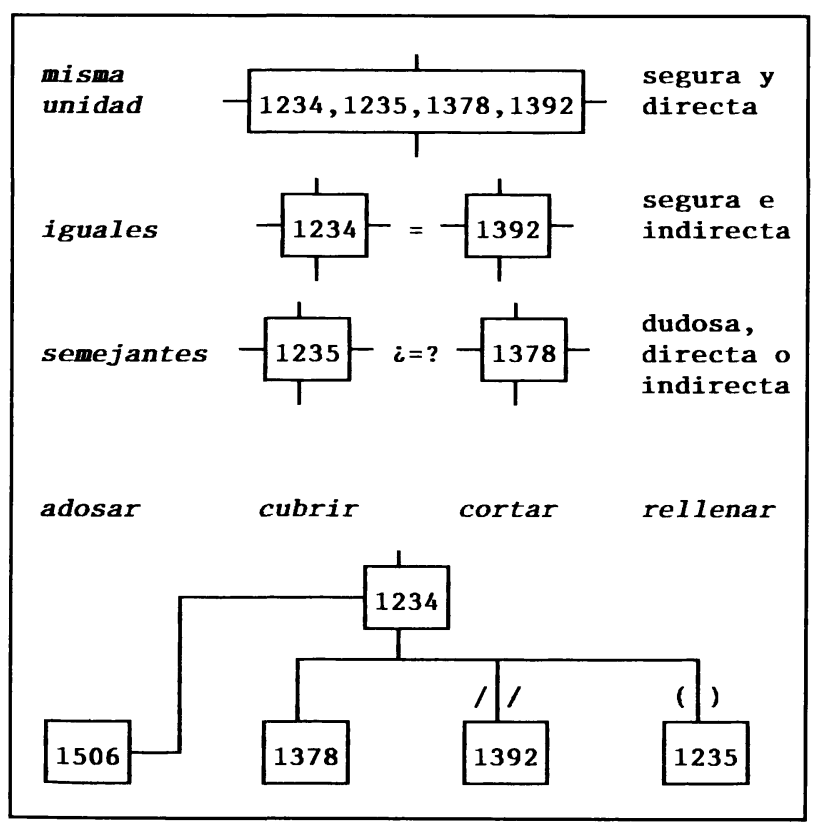

temporales posibles sólo son dos, la de coetaneidad y la de antero/posterioridad. La lectura sin embargo puede ofrecer distintas posibilidades en las que repercuten no sólo la dificultad que de por sí ofrezca el edificio, sino también la preparación del analista que la esté efectuando. Ante todo puede ser segura o dudosa; además de directa o indirecta -o sea constatándose el contacto entre los elementos o no-, lo que hace necesario un grafismo propio para el diagrama y supone un problema en la comprensión del vector temporal de coetaneidad. (Comparar con los conceptos geológicos DyH:5.7-13 y cap. 10).

La lectura, además, puede ser simple o compleja. En ocasiones cuando es compleja -o sea, con dos o varias acciones simultáneas entre dos o más elementos-, puede aconsejar usar un grafismo propio para construir el diagrama.

Los pasos de simplificación, correlación y periodización. Las estructuras.- El paso del diagrama de elemento a diagramas más complejos conlleva la previa reducción de las relaciones redundantes, suprimiendo las intermedias y reduciendo a una sola las relaciones complejas entre dos elementos, de modo que se simplifiquen lo más posible las líneas de relación.

El siguiente paso es la periodización o puesta en fase con que, a través de la cronología relativa obtenida por la situación en los diagramas de los elementos, horizontal y verticalmente, y de sus características, acciones que los relacionan, tipologías de sus materiales y datos de cronología absoluta, se deducen los períodos históricos en la vida del edificio.

Los elementos situados en línea, horizontalmente, se deben considerar pertenecientes a una misma estructura, que puede abarcar varias líneas consecutivas. Con esta correlación, de hecho otra reducción, se agrupan los elementos en unidades, redactándose, si la complejidad lo pide, fichas de estructuras en que la descripción de la unidad/estructura se sustituye por el listado de elementos correlativos que la componen. Esta etapa del proceso conlleva problemas de interpretación semejantes a los que 
teníamos con los elementos. Primero decidir si el salto diacrónico de un grupo de elementos a otro supone una estructura diferente o sólo una fase más en una estructura, en cuyo caso estaríamos sólo ante un problema de periodización. Al contrario puede ocurrir que elementos situados en línea pertenezcan a estructuras distintas pero coetáneas -estructuras contiguas unidas, por ejemplo, en un proceso posterior-. Las relaciones entre estructuras responden a parecido esquema que el explicado para los elementos y por lo tanto son similares sus procesos de descripción, lectura de relaciones, diagramación y periodización. Esta reconversión de los elementos en unidades estructurales es de gran importancia en el análisis arquitectónico del edificio, pues supone una síntesis: recuperar de nuevo, bajo un criterio genético e histórico, la unidad que habiamos perdido del edificio al dividirlo en elementos para facilitar su análisis (en contra de Bonelli:9).
La publicación de las conclusiones.- La complejidad del proceso de análisis estratigráfico suele ser tal que dificulta mucho la redacción de una memoria para la publicación o de uso interno. Es evidente que la memoria debe tener en cuenta no sólo la presentación sintética de los resultados, sino también facilitar la argumentación seguida hasta llegar a ellos y la documentación que permita contrastar el proceso seguido para rehacer sus pasos y poder, en su caso, criticar o corregir los resultados a que se ha llegado. Sólo así podrá considerarse que el proceso es científico. La principal dificultad es el de publicar las fichas de análisis de elementos, dado el trabajo que supondría pasarlas a limpio y el volumen a publicar. Para solucionar este problema proponemos sustituir su publicación por la de listados de elementos, conjuntamente con una planimetría completa donde estén diferenciados los elementos por sus números. Los campos de los listados deben ser los siguientes:

LISTADO DE ELEMENTOS

\begin{tabular}{||l|l|l|l|l|l|l||}
\hline \hline No & $\begin{array}{l}\text { Nombre } \\
\text { (descripcn. breve) }\end{array}$ & $\begin{array}{l}\text { Situación } \\
\text { (zona, plano) }\end{array}$ & $\begin{array}{l}\text { Periodo } \\
\text { (coetáneos) }\end{array}$ & Anterior a Posteor. a & Estructura \\
\hline \hline & & & & & & \\
\hline
\end{tabular}

También la documentación gráfica debe ser adecuada ofreciéndose, a ser posible, juegos de planos con el estado actual del edificio, planos complementarios de los listados con la diferenciación y numeración de los elementos y planos de síntesis de las estructuras (perspectivas, por ejemplo Coll y otros:fig. 4ss.) equivalentes a las conclusiones.

Depósito público.- La documentación original y los instrumentos utilizados -especialmente las fichas, pero también los planos de zonas, los croquis, fotografias, etc.deben depositarse en un centro de documentación público con suficientes garantías, donde se conserven y puedan ser consultados y utilizados.

Análisis arqueológico y diagnosis restauradora.- Este método no sólo tiene un interés histórico, sinouna aplicación directa en la restauración de construcciones históricas, donde puede llegar a revolucionar su filosofía. Los arquitectos italianos subrayan esa aplicación, similar a la de la estratigrafía en geología (Brogiolo 1988b:339ss.; Doglioni:233; DyH:1.7). El análisis elementopor elemento permite efectuar una diagnosis precisa y detallada de todo el edificio que concluya en una prescripción igualmente detallada; aunque debe tenerse en cuenta, como ya hemos dicho, que conceptualmente no son lo mismo y de hecho no tienen por qué coincidir exactamente elemento estratigráfico y elemento constructivo. Además permite al arquitecto considerar el edificio como un ente vivo en evolución y no como un "tipo" teórico fósil (en contra del erróneo concepto de Bonelli:6), y evaluar y medir la importancia que cada fase ha tenido en la construcción del edificio final.

\section{BIBLIOGRAFÍA}

APARICIO BASTARDO, J. A. 1991: Análisis de formas constructivas: aproximación al caso burgalés, II Jornadas Burgalesas de Historia. Burgos en la alta Edad Media, Burgos (1990), Burgos, 443-456.

BONELLI, R. 1986: Archeologia stratigrafica e storia dell' architettura, Architettura, Storia e Documenti, 2, 5-10.

BROGIOLO, G. P. (1988a): Archeologia dell'edilizia storica, Museo Civico Archeologico Como (sin año).

BROGIOLO, G. P. 1988b: Campionatura e obiettivi nell' analisi stratigrafica degli elevati, en FRANCOVICH y PARENTI 1988: 335-346.

CABALLEROZOREDA. L. 1987: El método arqueológico para la comprensión del edificio. Dualidad sustrato-estructura, Curso de mecánica y tecnología de los edificios antiguos, Colegio Oficial de Arquitectos de Madrid, 13-58.

CABALLERO ZOREDA, L. (coord.) (1992): Sobre el análisis arqueológico de construcciones históricas. La experiencia de Santa Eulalia de Mérida, la Torre de Hérculềs en La Conuña y $\mathrm{S}$. Pelayo de Arlanza, III Encuentros sobre Arqueologia y Patrimonio de Salobreña. Arqueología del monumento (en prensa). 


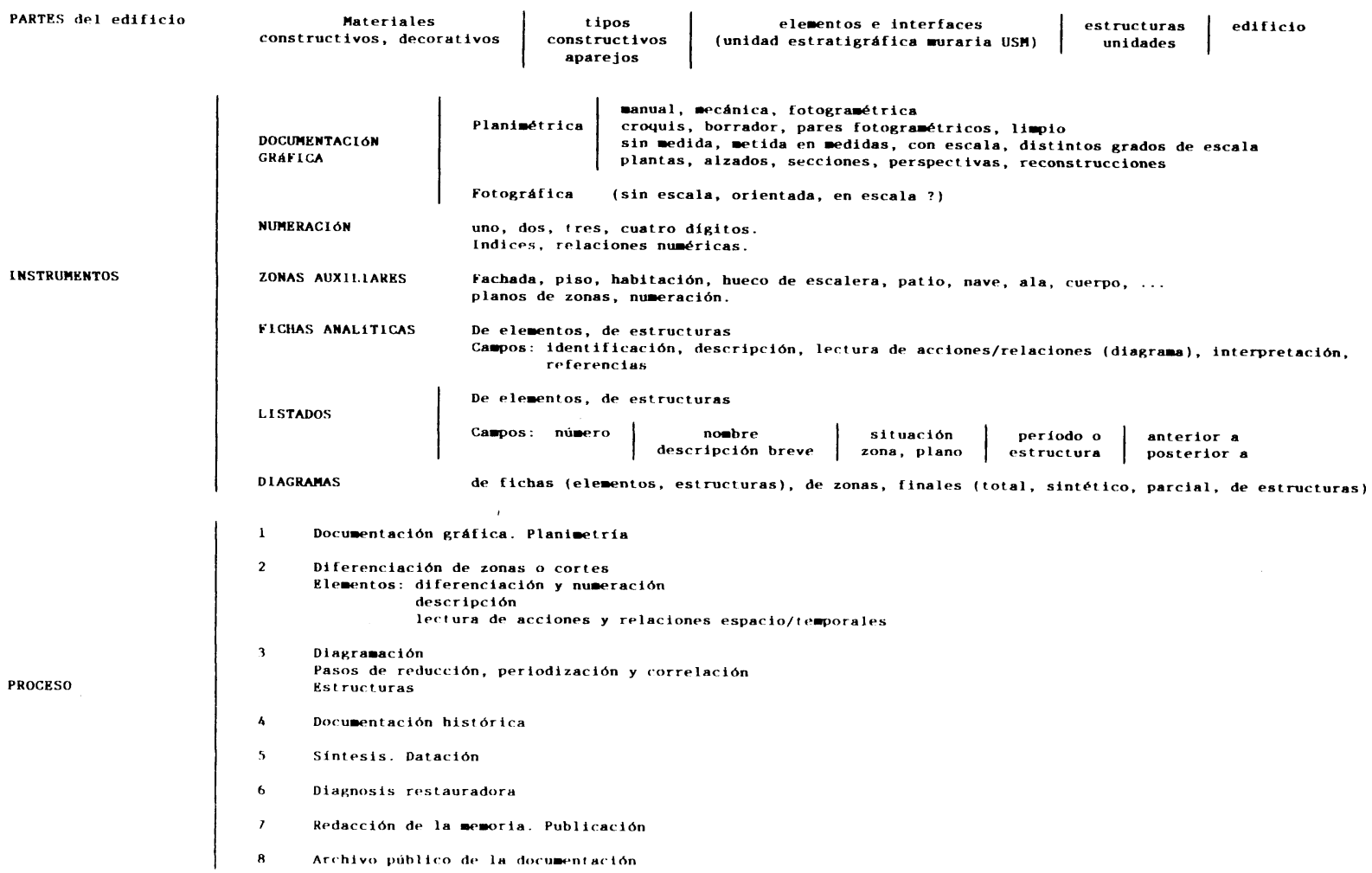

CABALLEROZOREDA, L., CÁMARA MUÑOZ,L.,LATORRE GONZÁLEZ-MORO, P. y MATESANZ VERA, P. (1991/1992): La iglesia prerrománica de S. Pedro el Viejo (Hortigüela, Burgos), Numantia (5,139-165).

CARANDINI, A. 1981: Storia della terra. Manualle dello scavo archeologico, Bari.

COLL CONESA, J., HUÉLAMO GABALDÓN, J. Mª y SOLIAS I ARIS, J. Ma 1992: L'edifici de la Inquisició de Cuenca. Avanç a la metodologia desenvolupada al seu estudi arqueoarquitectonic, en TRÓCOLI i SOSPEDRA 1992: 2, 77 ss.

DyH (1993): DABRIO, C. y HERNANDO, S., Estratigrafia, Departamento de Estratigrafia, Facultad de Ciencias Geológicas, Universidad Complutense de Madrid, Madrid (manuscrito).

DOGLIONI, F. 1988: La ricerca nelle struttura edilizia tra Archeologia stratigrafica e restauro architettonico, en FRANCOVICH y PARENTI 1988: 223-247.

FRANCOVICH, R. 1985: Contributi per l'unità delle discipline. Archeologia e restauro: da contiguità a unitarietà, Restauro and Città, 1, 11-20.

FRANCOVICH, R. 1988: Archeologia erestauro dei monumenti. Nota introduttiva, en FRANCOVICH y PARENTI 1988: 13-27.

FRANCOVICH, R. y PARENTI, A. (edit.) 1988: Archeologia e restauro dei monumenti, Consiglio Nazionale delle Ricerce. Università degli Studi di Siena, Florencia.
GALINDO I TORRES, J., MAURI I MARTI, A. y ROVIRATOVELLA, R. 1993: Les tècniques constructives del castell de Gelide, IV Congreso de Arqueologia Medieval Española, 2, 389 ss.

HARRIS, E. C. 1989: Principi di stratigrafia archeologica, La Nuova Italia Scientifica, Roma (1983).

HARRIS, E. C. 1991: Principios de estratigrafia arqueológica, Editorial Crítica, Barcelona (1989). Traducción I. García Trócoli.

HARRIS, E. C. 1992: Stratigraphy is the Matrix of Archaeology, en TRÓCOLI i SOSPEDRA 1992: 1, 84-104.

LÓPEZ I MULLOR, A; FIERRO I MACÍA, X. y CAIXAL I MATA, A. 1993: L'excavació a l'esglesia de Sant Jaume Sesoliveres (Igualada, L'anoia), Gala, 2, 259-276.

MANACORDA, D. 1989: Introduzione, en HARRIS 1989: 9-36.

MARINO, L. 1994: Il rilievo per il restauro. Ricognizioni, misurazioni, accertamenti, restituzioni, elaborazioni, Editore Ulrico Hoepli. Milán(1990). V. especialmente, 21. La stratigrafia degli elevati, 175-185.

PARENTI, R. 1985: La lettura stratigrafica delle murature in contesti archeologici e di restauro architettonico, Restauro \& Città , 2, 55-67

PARENTI, R. 1988a: La technique di documentazione per una lettura stratigrafica dell'elevato, en FRANCOVICH y PARENTI 1988: 249-279. 
PARENTI, R. 1988b: Sulla posibilità di datazione e di classificazione delle murature, en FRANCOVICH y PARENTI 1988: 280-304.

PRIETO VÁZQUEZ, G. 1987: Sobre el método Harris de excavación arqueológica, Carpetania. Revista del Museo de Santa Cruz, 145-154.
TRÓCOLI, I. G. y SOSPEDRA, R. (edit.) 1992: Harris Matrix. Recording systemes in Archaeology, El fil d'Ariadna, Lérida. Traducción I. G. Trócoli y S. MacName.
1. Intento no perder la relación con el método estratigráfico por excelencia, el geológico. Por ello he procurado ajustarme, en la definición de conceptos y terminología, al excelente manual de Dabrio y Hernando (DyH 1993), aunque en muchas ocasiones no haga referencia a él para no alargar más de lo necesario este texto.

2. Por abreviar no explicito otros principios no controvertidos, derivados de la geología (DyH: cap. 1), que se usan por costumbre en arqueología y que se aplican igual en el estudio histórico de la arquitectura; son los de la identidad tipológica, de los fragmentos incluidos, de la interdependencia de acciones y del actualismo y uniformismo.

3. Mal traducido al español por interfacies (Harris 1991, en cambio Harris 1992:105 n.4). Neologismo que traduce el término inglés interface, italiano interfaccia (Harris 1988), que sería intersuperficie, intercara o mejor, interfaz, interfaces, interfacial. A mi parecer interfacie es desafortunado por incluir facies, importante concepto geológico que significa características de las unidades geológicas y no las superficies de los estratos (Ver también Prieto; 148, n. 12).

4. Por definición no se puede confundir el estrato, elemento o unidad estratigráfica muraria con un elemento arquitectónico estructural(Parenti 1988a: 276; Marino: 175, 182). El elemento estratigráfico, básicamente histórico, puede coincidir o no con el estructural: la identidad no siempre se da.

5. Personalmente opino, como Parenti, que la definición de las acciones necesita mayor discusión hasta llegar a una normativa consensuada. Parenti (1988a: 269ss.) propone sólo cinco: se une, equivalente a nuestra igual a; se apoya, vertical, horizontalmente y rellenando sino existe corte; corta; rellena, sólo si existe corte; y cubre, para los revestimientos. Mis principales diferencias con él están en considerar posibles, al menos teóricamente, todas las acciones constructivas y en que unir es una acción constructiva que puede conllevar coetaneidad o antero/posterioridad.

\section{Publicación del Instituto Eduardo Torroja-CSIC}

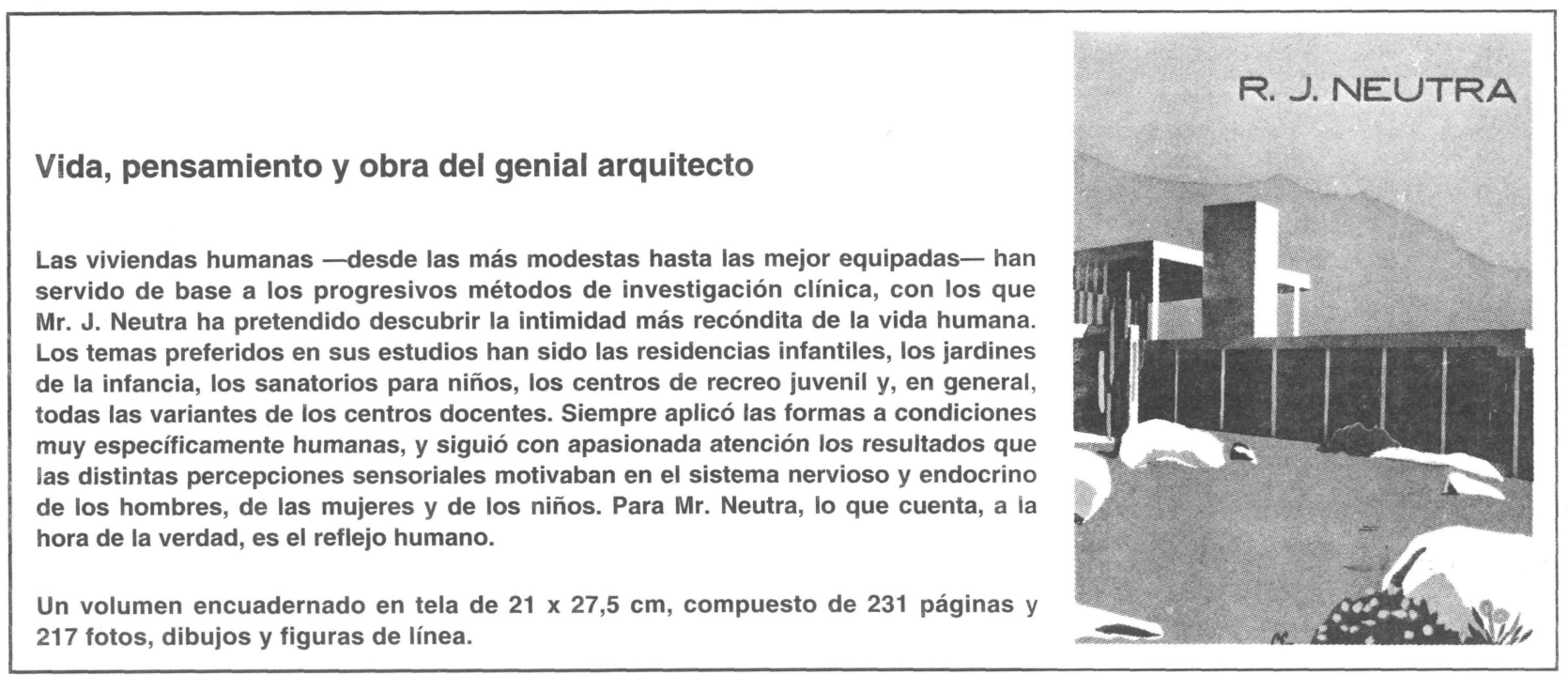

